\title{
$\int$

Patient compliance with new oral anticoagulants after major orthopaedic surgery: rivaroxaban and dabigatran compared with subcutaneous injection of fondaparinux

\author{
PAOLO DI BENEDETTO ${ }^{1}$, LUIGI VETRUGNO ${ }^{2}$, DANIA DE FRANCESCHI ${ }^{1}$, RENATO GISONNI ${ }^{1}$, \\ ARALDO CAUSERO ${ }^{1}$, GIORGIO DELLA ROCCA² \\ ${ }^{1}$ Department of Orthopaedic Surgery, University Hospital of Udine, Udine, Italy \\ 2 Department of Anesthesia and Intensive Care Medicine, University Hospital of Udine, Udine, Italy
}

\begin{abstract}
Purpose: the main purpose of our study was to compare patient compliance with the orally administered new oral anticoagulants (NOCs) dabigatran and rivaroxaban compared with subcutaneously injected fondaparinux after major orthopaedic surgery, and to assess patient preference for the oral $v s$ subcutaneous administration route.

Methods: prophylactic antithrombotic drug therapy with dabigatran (group D; GD, $\mathrm{n}=32$ patients), rivaroxaban (group R; GR, $\mathrm{n}=38$ patients) or fondaparinux (group F; GF, $n=30$ patients), to prevent deep vein thrombosis, was started immediately after surgery in 100 patients submitted to total hip arthroplasty.

Results: the patients had a mean age of $68.7 \pm 11$ years and $62 \%$ were female. In GD, $87.5 \%$ of patients indicated that they preferred oral intake of medications to subcutaneous injection (12.5\%). In GR, 84.2\% declared a preference for oral administration over subcutaneous injection (15.8\%). In GF, a surprisingly high proportion of patients $(73.3 \% ; \mathrm{p}<0.001)$ declared that they preferred subcutaneous administration of medications over the oral route $(26.7 \%)$. Overall, the rate of compliance with antithrombotic drug therapy was very high, at $99 \%$.
\end{abstract}

Conclusions: intake of the NOAs dabigatran and

\footnotetext{
Corresponding Author:

Luigi Vetrugno, MD

Department of Anesthesia and Intensive Care Medicine, University Hospital of Udine

Piazzale S. M. della Misericordia 15, 33100 Udine, Italy

E-mail: vetrugno.luigi@aoud.sanita.fvg.it
}

rivaroxaban following hospital discharge is entirely the responsibility of the patient; a high level of patient compliance with these drugs must therefore be demonstrated in order for them to become well accepted within the medical community. The results of this study showed a very high level of compliance both with orally and subcutaneously administered drugs.

Level of evidence: Level I, randomized clinical study.

Keywords: venous thrombosis, drug administration route, compliance, dabigatran, rivaroxaban, fondaparinux.

\section{Introduction}

Minimizing the incidence of deep vein thrombosis (DVT) and associated complications, such as pulmonary embolism (PE), remains an important challenge after major orthopaedic surgery (1-4). Fatal PE, although rare in patients receiving antithrombotic prophylaxis, has been widely described, occurring in 0.1 to $2 \%$ of cases (1). In the absence of prophylaxis, the incidence of DVT can be as high as $42-57 \%$ following total hip arthroplasty (THA) (1). Since their introduction in the 1980s, low molecular weight heparins (LMWHs) have become a gold standard in medical practice and the benchmark against which to compare new antithrombotic drugs $(5,6)$. LMWHs are obtained pharmacologically through the enzymatic depolymerization of unfractionated heparin, which reduces its molecular weight to a third. LMWHs have a better safety profile compared with unfractionated 
heparin due to their lower antithrombin and factor Xa inhibitory activities; another advantage is that they are given in fixed doses without the need for routine patient laboratory tests to determine the appropriate dose. The mandatory subcutaneous administration of LMWHs, on the other hand, is their biggest disadvantage and may reduce patient compliance. Indeed, it is widely thought that the ideal drug for the prevention of DVT would be a drug administered orally (7-9). Recently, a new class of drugs $(8,10-13)$ that are, indeed, endowed with this characteristic, has been introduced into clinical practice; these new oral anticoagulants (NOAs) are: rivaroxaban, a direct inhibitor of factor Xa with predictable pharmacokinetics and pharmacodynamics, which does not need to be dosed according to age, gender or body mass $(14,15)$; and dabigatran (16-19), a drug exhibiting a rapid onset and predictable anticoagulation activity.

Finally, another potent anti-thrombotic agent, fondaparinux, has also been introduced onto the market (20); an indirect specific inhibitor of factor $\mathrm{Xa}$, fondaparinux is completely eliminated by the kidneys and must therefore be used cautiously in patients with renal disease (20, 21). The disadvantage of fondaparinux, however, is that it can only be administered by subcutaneous injection.

In recent years, a number of randomized controlled trials have sought to verify the efficacy of these drugs $(5,22,23)$ and also to assess the incidence of DVT in patients treated with LMWHs, some studies comparing them with patients treated with NOAs and others comparing them with patients receiving fondaparinux or NOAs $(5,17,22-24)$. By contrast, very few studies have attempted to assess patient compliance with these treatments during the postoperative period following major orthopaedic surgery.

It is generally assumed that the oral route would be preferred by patients over subcutaneous injection, but this remains to be confirmed (7-9). The main purpose of our study was to evaluate patient compliance with oral intake of NOAs (dabigatran and rivaroxaban) as compared to subcutaneous injections of fondaparinux following major orthopaedic surgery. This study was conducted to explore the hypothesis that patient compliance with the orally administered NOAs dabigatran and rivaroxaban is superior to compliance with subcutaneously injected fondaparinux after major orthopaedic surgery.

\section{Methods}

\section{Study design}

This study was approved by the Ethics Committee of the "S. Maria della Misericordia" University Hospital of Udine (protocol number \#11568) and all the patients gave their written informed consent to participate. The patients were all submitted to elective prosthetic orthopaedic surgery, specifically a first total hip replacement.

\section{Patients}

Before surgery, the patients underwent a routine clinical evaluation, including the taking of a detailed medical history, a physical evaluation, routine laboratory tests, 12-lead electrocardiography, and chest radiography. The patient inclusion criteria were: age over 18 years and the willingness to participate, while the patient exclusion criteria were: revision surgery, current oral anticoagulant and/or antiplatelet therapy, or a creatinine clearance rate of less than $30 \mathrm{ml} / \mathrm{min}$.

\section{Interventions}

Before surgery, all patients underwent a venous Doppler examination of the legs to rule out DVT. Cardiovascular medications were left unchanged, except for angiotensin-converting enzyme inhibitors, which were stopped 1 day before surgery. Anesthesia was conducted by consultant anesthetists. Intrathecal injections were performed in the pre-operating room. Patients received either $10 \mathrm{mg}$ of hyperbaric bupivacaine (Angelini-A.C.R.A.F, Rome, Italy) or $10 \mathrm{mg}$ of isobaric levobupivacaine (Abbot S.r.l., Campoverde di Aprilia, Latina, Italy), at the anesthetist's discretion. All patients underwent anterior approach hip arthroplasty. For the procedure, the patient was placed in supine position. A longitudinal skin incision of about $8 \mathrm{~cm}$, two fingers distally and laterally to the anterior superior iliac spine, was performed, followed by incision of the fascia of the tensor fasciae latae. The rectus femoris was moved medially and the medius gluteus laterally. Once the joint was reached, capsulectomy was performed. After femoral neck resection and preparation of the acetabulum with a reamer, the acetabular cup was positioned. Then, the femur was broached and a trial component inserted. Once the correct positioning of the components had been checked, the final implant 


\section{Joints}

。

was inserted and the wound closed. Postoperative pain management consisted of oral oxycodone $10 \mathrm{mg}$ (BARD Pharmaceutics Ltd, Cambridge, UK) administered before and 12 hours after hip surgery, together with $10-15 \mathrm{mg} / \mathrm{kg}$ i.v. acetaminophen (Fresenius Kabi S.r.l, Verona, Italy), given every 6 hours for 72 hours postoperatively. After surgery, the patients were randomly assigned by computerized randomization (Microsoft Office Excel 2010 for Windows, Microsoft Corporate, Mountain View CA, USA) to one of three groups: the Group D (GD) patients received oral dabigatran (Pradaxa ${ }^{\mathcal{C}}$ Boehringer Ingelheim International $\mathrm{GmbH}$, Ingelheim am Rhein, Germany) at the recommended dose of $110 \mathrm{mg}$, between 1 and $4 \mathrm{~h}$ after the end of the surgery, followed by single daily doses of $220 \mathrm{mg}$ for the subsequent 35 days; the Group R (GR) patients received oral rivaroxaban $\left(\right.$ Xarelto $^{\odot}$, Bayer Pharma AG, Berlin, Germany) at the dose of $10 \mathrm{mg}$ once a day starting on the day of the surgery and continuing for the next five weeks; the Group F (GF) patients received a subcutaneous injection of fondaparinux (Arixtra ${ }^{\complement}$ Aspen Pharma Trading Limited, Dublin, Ireland) at a dose of $2.5 \mathrm{mg}$ once a day. The dose of fondaparinux was adjusted taking into account renal function evaluated on the basis of serum creatinine levels. The data collected during the study period included details of the surgery and anesthesia and any complications, also relating to the thromboprophylaxis, arising during the entire length of stay (LOS). Thirty days after surgery, all the patients (GR, GD, GF) underwent a hip X-ray evaluation followed by a clinical examination. At that time, a structured questionnaire, designed by the investigators (Appendix 1) was filled in by each patient.

\section{Outcome measures}

The primary endpoint of the study was proper use of (compliance with) the prescribed doses of dabigatran, rivaroxaban and fondaparinux. Because of the wellknown interaction between the NOAs and amiodarone, intake of the latter was also recorded. If patients did not take a daily dose, this was recorded as "incorrect drug intake adherence". Two of the Authors of the present study (PDB and DDF) scored the questionnaires and compiled the corresponding charts. The two investigators discussed each individual case with the aim of reaching a common assessment of the patient's compliance. In cases of disagreement, the lowest agreement was recorded. The secondary endpoints of this study were the following: the occurrence of symptomatic DVT or PE and bleeding events, LOS, hospital readmission, outpatient clinic visits, and any interventions performed as a result of complications.

\section{Statistical analysis}

For the entire sample, the following were calculated: mean and standard deviation for age, and frequency and percentage values for gender, number of patients

\section{Appendix 1}

Have you taken the dabigatran (tablets), rivaroxaban (tablets) or fondaparinux (vials) as prescribed? $\quad$ Yes $\square$ No $\square$ If not, indicate how many more or less tablets/vials more or less you

took:

Did you have any gastrointestinal disorders (nausea, vomiting, diarrhea, stomach pain ...) after taking dabigatran, rivaroxaban or fondaparinux? Yes $\square$ No $\square$

If yes, specify the type of disorder:

Have you taken any other drugs while using dabigatran, rivaroxaban or fondaparinux?

(in particular amiodarone)?

If yes, what kind of drugs?:

Yes $\square$ No $\square$

Did you experience any hemorrhagic manifestations (bleeding from the nose, gums, bruising, etc.) while taking dabigatran, rivaroxaban or fondaparinux?

Yes $\square$ No $\square$

If yes, specify what kind of hemorrhagic manifestations:

Did you consume any alcohol during the treatment with dabigatran, rivaroxaban or fondaparinux?

Yes $\square$ No $\square$

Do you think that oral administration of a drug is preferable to administration by subcutaneous injection? Yes $\square$ No $\square$ If yes, specify why: 
taking each drug studied, and patient compliance. The chi square test or Fisher's exact test was used to assess associations between the drug used and compliance. The Shapiro-Wilk normality test was used to assess the distributions in each group, and the Kruskall Wallis test was used to evaluate differences in LOS between the three groups. Lebel et al. evaluated patient compliance with a NOC following major orthopaedic surgery, and found it to be $66.1 \%$ (25). On the basis of their study, we deduced that a simple size of 91 patients, assuming a drop-out rate of $10 \%$, would have the power to detect a statistically significant difference. Microsoft Excel was used to create an electronic database of all the collected data, and $\mathrm{p}$ values $\leq 0.05$ were considered statistically significant. STATA 13 for Windows (Stata Corp LP, College Station, TX, USA) software was used for all the statistical analyses.

\section{Results}

During the study period (March 2013-February 2015), we enrolled a total of 112 patients undergoing total hip replacement surgery. Of these, 12 were lost during the follow-up period. Of the remaining 100 patients, 62 were females and 38 were males. A summary of the patient characteristics, coexisting diseases, current medication and LOS data are reported in Table 1.

The patients had a mean age of $68.7 \pm 11$ years, range $36-86$ years; $62 \%$ were females. The number of patients included in each group were as follows: dabigatran, $n=32$; rivaroxaban, $n=38$; fondaparinux, $n=30$. We found no significant between-group differences in gender or age. The power of the study reached our target of $90 \%$. In the overall population, the rate of compliance with antithrombotic therapy was very high, reaching $99 \%$. No patient exhibited "over compliance", i.e. taking more than one daily dose (noncompliance). In the course of the entire study period, only one patient forgot to take just one dose of rivaroxaban. In GD, $87.5 \%$ of patients indicated a preference for oral administration of a drug over subcutaneous injection $(12.5 \%)$.

Similarly, $84.2 \%$ of patients in GR indicated that they preferred oral administration over subcutaneous injection $(15.8 \%)$. In GF, on the other hand, in which the drug was administered by subcutaneous injection, a
Table 1. Patient demographic data, coexisting diseases, medications and length of hospital stay.

\begin{tabular}{ll}
\hline Number of patients: & 100 \\
Age (yrs) (Mean \pm SD) & $68.7 \pm 11$ \\
Men/Women & $38 / 62$ \\
\hline Coexisting diseases & \\
Smokers & $(16 \%)$ \\
Hypertension & $(41 \%)$ \\
Diabetes & $(8 \%)$ \\
COPD & $(3 \%)$ \\
Previous AMI & $(5 \%)$ \\
Previous CABG/PTE & $(3 \%)$ \\
Medications & $(12 \%)$ \\
B-blockers & $(38 \%)$ \\
ACE inhibitor/AR antagonist & $(18 \%)$ \\
ASA & $(28 \%)$ \\
Statins & $(1 \%)$ \\
TNG & $12.29 \pm 7.45$ \\
Length of stay (days) & \\
\hline
\end{tabular}

NOTE. The data are presented as percentage values, which also correspond to patient numbers since the sample comprised 100 patients, and as mean values \pm standard deviations.

Abbreviations: ACE, angiotensin-converting enzyme; AMI, acute myocardial infarction; $\mathrm{AR}$, angiotensin receptor; $\mathrm{CABG}$, coronary artery bypass grafting; COPD, chronic obstructive pulmonary disease; PTE, percutaneous thromboendarterectomy.

surprisingly high number of patients $(73.3 \%)$ reported a preference for this route over oral administration $(26.7 \%)$ (Fig. 1). In other words, the patients who took medication via the oral route considered this to be preferable to subcutaneous injection. In the same way, those receiving subcutaneous injections also pre-

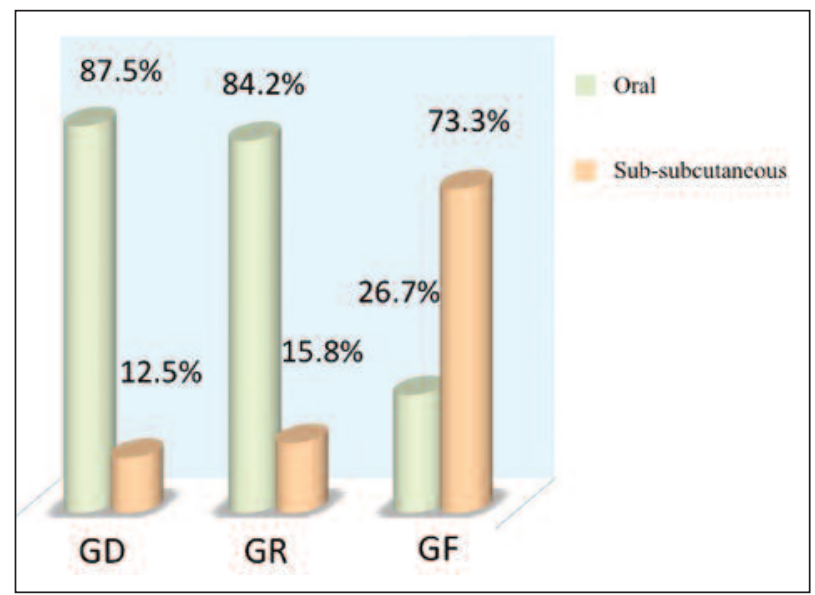

Figure 1. Patients' preference (\%) for oral administration or subcutaneous injection (GD: dabigatran group; GR: rivaroxaban group; GF: fondaparinux group). 


\section{oints}



ferred their assigned route of administration. Indeed, when asked "Do you think that oral administration of a drug is preferable to administration by subcutaneous injection?" a significantly higher frequency of patients treated with fondaparinux $(73.3 \% ; \mathrm{p}<0.001)$ compared to those taking dabigatran $(12.5 \%)$ or rivaroxaban $(15.8 \%)$ replied that oral administration is not preferable to subcutaneous injection.

Ten percent of the patient population exhibited gastrointestinal disorders during the course of the treatment: $15 \%$ of GD (n=5), $5 \%$ of GR $(n=2)$, and $10 \%$ of GF ( $n=3)$; instead, $6 \%$ of the entire sample exhibited minor bleeding events, without the need for hospitalization: $3 \%$ of GD ( $n=1), 2 \%$ of GR $(n=1)$, and $13 \%$ of GF ( $\mathrm{n}=4$ ) (Fig. 2). During the study period, $40 \%$ of patients declared that they were on other medication: $50 \%$ of GD ( $n=16), 36 \%$ of GR $(n=14)$, and $33 \%$ of GF $(n=10)$. No patients required treatment with amiodarone. Twelve percent of the patients also drank alcohol (1 or 2 glasses of wine a day): 6 patients in GD, 2 patients in GR and 4 patients in GF. No associations were found between study drug administration and the occurrence of gastrointestinal disorders (Fisher's test; $\mathrm{p}=0.380$ ), and no associations were found between study drug administration and the use of other medications during the study period $\left(\mathrm{X}^{2}\right.$ test; $\left.\mathrm{p}=0.359\right)$. We did not find any statistically significant association between the drug used and bleeding events (Fisher's test; $\mathrm{p}=0.183$ ) or between the drug

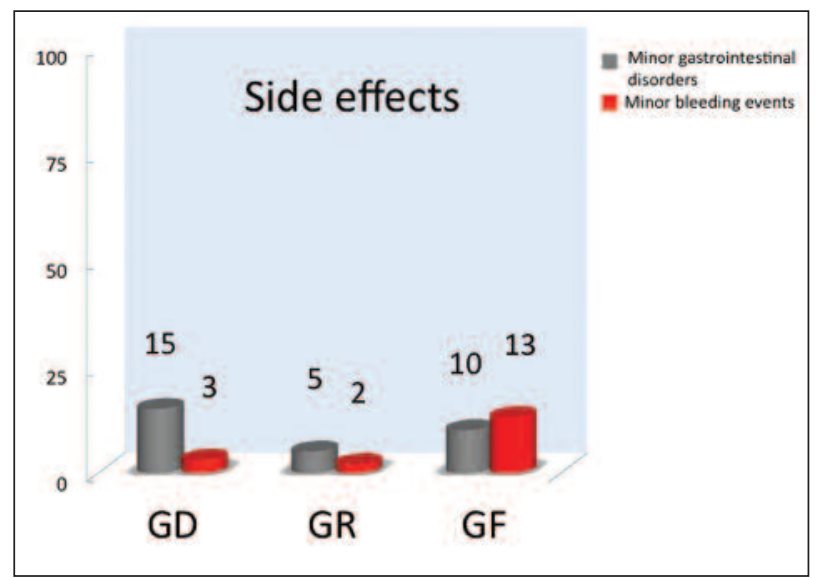

Figure 2. Incidence of minor side effects: $10 \%$ gastrointestinal disorders (15 patients in GD, 5 patients in GR, and 10 patients in GF); $6 \%$ presenting minor bleeding events 3 (3 patients in GD, 2 patients in GR, and 13 patients in GF) (GD: dabigatran group; GR: rivaroxaban group; GF: fondaparinux group). used and alcohol consumption (Fisher's test; $\mathrm{p}=0.468)$.

None of the patients developed symptomatic DVT and/or PE. Finally, we analyzed and compared LOS between the three different groups. In GD, the mean LOS was $7.7 \pm 2.6$ days, ranging from a minimum of 4 to a maximum of 16 days; in GR, the mean LOS was $6.8 \pm 2.1$ days (range: 4 to 12 days); and in GF the mean LOS was $7.8 \pm 2.3$ days (range: 5 to 15 days). There was no association between drug and LOS $(\mathrm{p}=0.166)$.

\section{Discussion}

The main finding of the present study was the very high rate of compliance with the antithrombotic therapy in our patient population: $99 \%$. In the groups receiving NOAs (dabigatran and rivaroxaban), oral administration was reported to be the preferred intake route by $87.5 \%$ and $84.2 \%$ of patients, respectively. Surprisingly, a high percentage of patients receiving fondaparinux by subcutaneous injection (73.3\%) instead considered this route preferable to oral administration. In other words, all the groups preferred their own assigned mode of drug intake, be it via the oral route or via subcutaneous injection.

Anticoagulant prophylaxis with LMWHs has been considered a gold standard for many years, and today represents an important benchmark against which to compare new antithrombotic drugs $(1,12)$. The main disadvantage of treatment with LMWHs is that it requires subcutaneous administration, reported by patients to be uncomfortable and inconvenient; consequently, they sometimes interrupt the therapy $(7,8)$. In short, administration by subcutaneous injection may reduce patient compliance. This would, therefore, also be expected to be an inherent problem associated with the use of fondaparinux.

In summary, we found that the NOAs investigated are as effective as LMWHs and show no differences in compliance when compared with subcutaneous injection of fondaparinux. Furthermore, compliance with these NOAs was not associated with the use of any other drugs or with alcohol consumption. In support of our findings, little or no interaction has been reported in the literature between dabigatran and acetaminophen or non-steroidal anti-inflammatory drugs, which are widely used in the postoperative period (7, 
13, 26, 27). The most recent version of the American College of Chest Physicians guidelines, published in 2012, recommends thromboprophylaxis with anticoagulants for a minimum of 10 to 14 days in patients undergoing THA (grade 1B), which should be extended in the outpatient period for $\leq 35$ days from the day of surgery (grade 2) (4). Randomized controlled trials have recently demonstrated that these novel agents are at least as effective as LMWHs and are not associated with an increased incidence of clinically significant bleeding $(12,28,29)$. Thus, dabigatran, rivaroxaban and fondaparinux - the new inhibitors of thrombin or factor $\mathrm{Xa}$ - represent a valid alternative to LMWHs. Huisman et al. showed that enoxaparin is less effective than rivaroxaban (29), while another meta-analysis comparing the efficacy and safety of dabigatran with enoxaparin suggested that these medicines show similar efficacy and associated bleeding rates in patients undergoing hip arthroplasty (30). Thus, anticoagulation therapy options should now include the NOAs dabigatran and rivaroxaban, as well as fondaparinux, even though some argue that LMWHs should be preferred on the basis of their longer clinical track record compared with the new alternatives (grade 2) $(5,7)$.

Regarding the correct timing of administration, the results of our study agree with the 2014 Surgical Care Improvement Project (7), which stated that patients undergoing THA should receive appropriate prophylaxis (which includes the new oral anticoagulants) within 24 hours before surgery. In this regard, it is very important to select the most appropriate thromboprophylaxis by balancing the risk of bleeding versus the prevention of venous thromboembolism (VTE). Slower, and therefore reduced (by 20-25\%), absorption of dabigatran has been described when it is coadministered with proton pump inhibitor (PPI) drugs (26). The kinetic profile of dabigatran is widely known: the maximum plasma concentration ( $\mathrm{t}$ max) is reached 2 hours after administration. The $t$ max could be delayed to 3-4 $\mathrm{h}$ after administration if dabigatran is swallowed with food or taken with PPI drugs or in the presence of stress conditions that slow gastric emptying (26). Its plasma half-life is $12-14 \mathrm{~h}$, so it is not dependent on dose protocols $(13,27)$. The steady state is reached in 2-3 days. The maximum effect on coagulation parameters coincides with the maximum plasma concentration of the drug (26). Some coagulation laboratory parameters are affected by its adminis- tration: the activated partial thromboplastin time (aPT'T) ratio was found to increase linearly with drug dose, as was the thrombin time, with the maximum alteration observed 2-4 $\mathrm{h}$ after intake (27). On the other hand, it is not known whether dabigatran administration alters the thromboelastogram (TEG) trace. The results of studies comparing dabigatran and enoxaparin on the basis of the evidence from REMODEL (the prevention of venous thromboembolism after total knee replacement trial) and RENOVAT (the prevention of venous thromboembolism after total hip replacement trial) (14-30) have shown that dabigatran has the same efficacy as a single administration of $40 \mathrm{mg}$ of enoxaparin; instead, REMOBILIZE (the prevention of venous thromboembolism after total knee arthroplasty trial) showed that dabigatran could meet the criteria of non-inferiority to a $30 \mathrm{mg}$ twice daily dose of enoxaparin. The pharmacological profile of rivaroxaban has also been extensively studied $(14,31,32)$. For example, rivaroxaban has been demonstrated to reach its maximum effect after just 2 to $4 \mathrm{~h}(31,32)$ and, as with dabigatran, its absorption is delayed by food intake, resulting in less inter-individual variability $(31,32)$. The half-life of rivaroxaban is 7-11 $\mathrm{h}$ and no significant differences have been observed between the effect of the first dose and once steady state is reached (14). The principal route of rivaroxaban excretion is via the kidneys; as a consequence, rivaroxaban is not recommended in patients with a creatinine clearance rate $<30 \mathrm{ml} / \mathrm{min}$ (14). However, rivaroxaban can be used in patients with cirrhosis of the liver in the absence of coagulopathy (14, 15); rivaroxaban shows good anti-Xa activity, while the aPTT ratio is less sensitive to rivaroxaban than the prothrombin time is. As in the case of dabigatran, it is not known how TEG traces are modified by rivaroxaban. The clinical use of rivaroxaban has been validated by the Phase III RECORD (Regulation of Coagulation in Orthopaedic Surgery to Prevent Deep Vein Thrombosis and Pulmonary Embolism) program $(15,33)$. Its use has thus been approved by the European Union and also in the USA for VTE prophylaxis in patients undergoing elective hip surgery (15). The third drug for DVT prophylaxis investigated in the present study was fondaparinux, administered by subcutaneous injection. One limitation associated with the use of this drug is the need to ascertain the presence of appropriate plas- 
ma levels of antithrombin. The usual prophylactic dose for DVT is $2.5 \mathrm{mg} /$ day via a single subcutaneous administration (20), although the dose can also be split into several daily administrations. In healthy subjects, the anti-Xa peak is obtained 2 hours post subcutaneous administration (34). Fondaparinux is well absorbed and has a half-life of 17 hours, permitting a once-daily injection (20). The liver metabolizes fondaparinux, by desulfation and/or depolymerization, into fragments of lower molecular weight and reduced biological activity. The steady state is achieved after 2 days of single daily administrations. Renal clearance of active metabolites eliminates about $10 \%$ of the administered dose, while renal excretion of active and nonactive metabolites eliminates $40 \%$ of the dose (35). Dose adjustment is recommended in the presence of a creatinine clearance rate $<50 \mathrm{ml} / \mathrm{min}$ (35). In the present study, no major side effects of fondaparinux therapy occurred; in particular, no bleeding events were recorded at 30 days. Surprisingly, in our population, no differences in patient compliance were detected between the subcutaneous route of administration (used for fondaparinux) and the oral route (used for rivaroxaban and dabigatran), constituting a novel finding. However, the results of our study are in line with those published by Lebel et al., who assessed compliance with oral thromboprophylaxis following total hip replacement and the possible consequences of noncompliance (25).

Those Authors reported a compliance rate of $66.1 \%$. Previous studies, focusing on use of anticoagulants in longer treatments, secondary prevention, or curative treatments for known pathologies, have shown compliance rates ranging from 31 to $83 \%$ (6-29); furthermore, unsurprisingly, compliance is observed to diminish over time $(1,11)$. To the best of our knowledge, no other studies have specifically evaluated patient compliance with different drugs administered by different routes - i.e. oral administration versus subcutaneous injection. Oral administration is generally considered to be the preferred route, being fast and simple compared to subcutaneous injection $(7,8)$, but more data is needed. The main limitations of our study need to be underlined. First of all, the factors influencing patient compliance may not only be the type of administration route used for the drug in question, but also by the patient's inclination and ability to look after his own health. Similarly, compliance may reflect the degree to which a person has been educated to follow rules. However, these aspects were not evaluated in this study. In addition, this study was a single-center explorative study, and therefore presented intrinsic limitations; nevertheless, the findings in our population were strong enough to reveal certain behaviors/attitudes regarding treatment compliance. In conclusion, the present study evaluated patient compliance with dabigatran and rivaroxaban treatment (bearing in mind that the patient is fully responsible for the administration of these NOAs following hospital discharge). A very high compliance rate was obtained, confirming the potential of these drugs to replace LMWHs as the gold standard for antithrombotic prophylaxis in medical practice. We also assessed patient compliance with fondaparinux therapy administered by subcutaneous injection and again found excellent compliance rates in our patient population. The patients in our study provided positive feedback about the treatments (their convenience, comfort, and intake simplicity, etc.), both with NOAs (dabigatran and rivaroxaban) and with fondaparinux. Surprisingly, neither administration route was revealed to be "superior" to the other. Finally, no major side effects or complications were observed caused by the co-administration of antithrombotic prophylaxis with other drug therapies or its association with moderate alcohol consumption.

\section{References}

1. Kinov P, Tanchev PP, Ellis M, et al. Antithrombotic prophylaxis in major orthopaedic surgery: an historical overview and update of current recommendations. Int Orthop. 2014;38: 169-175.

2. Markovic-Denic L, Zivkovic K, Lesic A, et al. Risk factors and distribution of symptomatic venous thromboembolism in total hip and knee replacements: prospective study. Int Orthop. 2012;36:1299-1305.

3. Eikelboom JW, Karthikeyan G, Fagel N, et al. American Association of Orthopedic Surgeons and American College of Chest Physicians guidelines for venous thromboembolism prevention in hip and knee arthroplasty differ: what are the implications for clinicians and patients? Chest. 2009;135:513520.

4. Falck-Ytter Y, Francis CW, Johanson NA, et al. Prevention of VTE in orthopedic surgery patients: Antithrombotic Therapy and Prevention of Thrombosis, 9th ed: American College of Chest Physicians Evidence-Based Clinical Practice Guidelines. Chest. 2012;141(2 Suppl):e278S-325S.

5. Johanson NA, Lachiewicz PF, Lieberman JR, et al. American academy of orthopaedic surgeons clinical practice guideline 
on. Prevention of symptomatic pulmonary embolism in patients undergoing total hip or knee arthroplasty. J Bone Joint Surg Am. 2009;91:1756-1757.

6. Rachidi S, Aldin ES, Greenberg C, et al. The use of novel oral anticoagulants for thromboprophylaxis after elective major orthopedic surgery. Expert Rev Hematol. 2013;6: 677-695.

7. Messerschmidt C, Friedman RJ. Clinical experience with novel oral anticoagulants for thromboprophylaxis after elective hip and knee arthroplasty. Arterioscler Thromb Vasc Biol. 2015;35:771-778.

8. Klauser W, Dütsch M. Practical management of new oral anticoagulants after total hip or total knee arthroplasty. Musculoskelet Surg. 2013;97:189-197.

9. Wong A, Kraus PS, Lau BD, et al. Patient preferences regarding pharmacologic venous thromboembolism prophylaxis. J Hosp Med. 2015;10:108-111.

10. Bass AR. Using new oral anticoagulants in patients undergoing major orthopedic surgery. Curr Rheumatol Rep. 2015;17:25. doi: 10.1007/s11926-015-0498-z.

11. van der Veen L, van Raay JJ, Gerritsma-Bleeker CL, et al. Direct treatment comparison of DAbigatran and RIvaroxaban versus NAdroparin in the prevention of venous thromboembolism after total knee arthroplasty surgery: design of a randomised pilot study (DARINA). BMJ Open. 2013;24;3. pii:e002218.doi:10.1136/bmjopen-2012-002218.

12. Prisco D, Cenci C, Silvestri E, et al. Pharmacological prevention of venous thromboembolism in orthopaedic surgery. Clin Cases Miner Bone Metab. 2014;11:192-195.

13. Eriksson BI, Quinlan DJ, Weitz JI. Comparative pharmacodynamics and pharmacokinetics of oral direct thrombin and factor xa inhibitors in development. Clin Pharmacokinet. 2009;48:1-22.

14. Perzborn E, Roehrig S, Straub A, et al. Rivaroxaban: a new oral factor $\mathrm{Xa}$ inhibitor. Arterioscler Thromb Vasc Biol. 2010;30:376-381.

15. Gulseth MP, Michaud J, Nutescu EA. Rivaroxaban: an oral direct inhibitor of factor Xa. Am J Health Syst Pharm. 2008; 65:1520-1529.

16. Postma MJ, Kappelhoff BS, van Hulst M, et al. Economic evaluation of dabigatran etexilate for the primary prevention of venous tromboembolic events following major orthopedic surgery in the Netherlands. J Med Econ. 2012;15:878-886.

17. Mrozik D, Jackiewicz A, Krzeminski M. Dabigatran vs. low molecular weight heparin in preventing venous thromboembolism after elective hip and knee arthroplasty: evaluation of selected clinical parameters. Pol Orthop Traumatol. 2012; 77:111-114

18. Burness CB, McKeage K. Dabigatran etexilate: a review of its use for the prevention of venous thromboembolism after total hip or knee replacement surgery. Drugs. 2012;72:963986.

19. Wolowacz SE, Roskell NS, Plumb JM, et al. Efficacy and safety of dabigatran etexilate for the prevention of venous thromboembolism following total hip or knee arthroplasty. A meta-analysis. Thromb Haemost. 2009;101:77-85.

20. Bergqvist $D$. Review of fondaparinux sodium injection for the prevention of venous thromboembolism in patients undergoing surgery. Vasc Health Risk Manag. 2006;2:365-370.
21. Turpie AG. Fondaparinux: a Factor Xa inhibitor for antihrombotic therapy. Expert Opin Pharmacother. 2004;5: 1373-1384.

22. Januel JM, Chen G, Ruffieux C, et al. Symptomatic in-hospital deep vein thrombosis and pulmonary embolism following hip and knee arthroplasty among patients receiving recommended prophylaxis: a systematic review. JAMA. 2012;307: 294-303.

23. Memtsoudis SG, Besculides MC, Gaber L, et al. Risk factors for pulmonary embolism after hip and knee arthroplasty: a population-based study. Int Orthop. 2009;33:1739-1745.

24. Lazo-Langner A, Fleet JL, McArthur E, et al. Rivaroxaban vs. low molecular weight heparin for the prevention of venous thromboembolism after hip or knee arthroplasty: a cohort study. J Thromb Haemost. 2014;12:1626-1635.

25. Lebel B, Malherbe M, Gouzy S, et al. Oral thromboprophylaxis following total hip replacement: the issue of compliance. Orthop Traumatol Surg Res. 2012;98:186-192.

26. Blech S, Ebner T, Ludwig-Schwellinger E, et al. The metabolism and disposition of the oral direct thrombin inhibitor, dabigatran, in humans. Drug Metab Dispos. 2008;36:386-399.

27. Stangier J, Rathgen $\mathrm{K}$, Stähle $\mathrm{H}$, et al. The pharmacokinetics, pharmacodynamics and tolerability of dabigatran etexilate, a new oral direct thrombin inhibitor, in healthy male subjects. Br J Clin Pharmacol. 2007;64:292-303.

28. Feng W, Wu K, Liu Z, et al. Oral direct factor Xa inhibitor versus enoxaparin for thromboprophylaxis after hip or knee arthroplasty: systemic review, traditional meta-analysis, doseresponse meta-analysis and network meta-analysis. Thromb Res. 2015;136:1133-1144.

29. Huisman MV, Quinlan DJ, Dahl OE, et al. Enoxaparin versus dabigatran or rivaroxaban for thromboprophylaxis after hip or knee arthroplasty: results of separate pooled analyses of phase III multicenter randomized trials. Circ Cardiovasc Qual Outcomes. 2010;3:652-660.

30. Friedman RJ, Dahl OE, Rosencher N, et al. Dabigatran versus enoxaparin for prevention of venous thromboembolism after hip or knee arthroplasty: a pooled analysis of three trials. Thromb Res. 2010;126:175-182.

31. Lang D, Freudenberger C, Weinz C. In vitro metabolism of rivaroxaban, an oral, direct factor Xa inhibitor, in liver microsomes and hepatocytes of rats, dogs, and humans. Drug Metab Dispos. 2009;37:1046-1055.

32. Weinz C, Schwarz T, Kubitza D, et al. Metabolism and excretion of rivaroxaban, an oral, direct factor $\mathrm{Xa}$ inhibitor, in rats, dogs, and humans. Drug Metab Dispos. 2009;37:1056-1064.

33. Lassen MR, Gent M, Kakkar AK, et al. The effects of rivaroxaban on the complications of surgery after total hip or knee replacement: results from the RECORD programme. J Bone Joint Surg Br. 2012;94:1573-1578.

34. Samama MM, Gerotziafas GT. Evaluation of the pharmacological properties and clinical results of the synthetic pentasaccharide (fondaparinux). Thromb Res. 2003;109:1-11.

35. Delavenne X, Zufferey P, Nguven P, et al. Pharmacokinetics of fondaparinux $1.5 \mathrm{mg}$ once daily in a real-world cohort of patients with renal impairment undergoing major orthopaedic surgery. Eur J Clin Pharmacol. 2012;68:1403-1410. 